\title{
Results of the workshop on strategies and tools for administrators of the territory of the italian alpine space for the shallow geothermal systems - GRETA Project - Near-surface Geothermal Resources in the Territory of the Alpine Space
}

\section{Risultati del workshop su strategie e strumenti per gli amministratori del territorio dello spazio alpino italiano per gli impianti che sfruttano la geotermia di bassa profondità - Progetto GRETA - Near-surface Geothermal Resources in the Territory of the Alpine Space}

\author{
Ilaria Stringa, Alessandro Baietto - GDP Consultants,Via Vittorio Amedeo 6 - 10121, Torino, Italy - baietto@gdpconsultants.eu \\ Francesco Spinolo - Regione Lombardia, U.O. Sviluppo Sostenibile e Risorse dell'Ambiente, 20124 Milano, Italy \\ Kai Zoesseder - Technical University of Munich (TUM) - Chair of Hydrogeology, 80333, Muenchen, Germany \\ Joerg Prestor - Geological Survey of Slovenia (GEOZS) - Department for groundwater and hydrogeology, 1000, Ljubljana, Slovenia \\ Simona Pestotnik - Geological Survey of Slovenia (GEOZS) - Department for groundwater and hydrogeology, 1000, Ljubljana, Slovenia \\ Doris Rupprecht - Geological Survey of Austria (GBA) - Main department of applied geosciences, A-1030 Wien, Austria \\ Pietro Capodaglio - ARPA Valle d'Aosta, 11020 St. Christophe (AO), Italy \\ Alessandro Casasso, Simone Della Valentina, Arianna Bucci - Politecnico di Torino - DIATI, 10129, Torino, Italy \\ Pietro Zambelli, Valentina D’Alonzo - Accademia Europea Bolzano - Institute for Renewable Energy, 39100, Bolzano, Italy \\ Carlo Enrico Cassani - Regione Lombardia - U.O. Risorse idriche, 20124 Milano, Italy \\ Stefania Ghidorzi- Infrastrutture Lombarde S.p.a, 20124, Milano, Italy
}

Keywords: geothermal heat pumps, GRETA Project, Alpine Space, administrative procedures, harmonization.

Parole chiave: pompe di calore geotermiche, Progetto GRETA, Spazio Alpino, procedure amministrative, armonizzazione.

\section{La geotermia di bassa profondità e il progetto GRETA}

I sistemi a pompa di calore che utilizzano la geotermia di bassa profondità (altrimenti nota come a "bassa entalpia") per il riscaldamento ed il raffrescamento degli edifici rappresentano una tecnologia efficiente e vantaggiosa che può contribuire significativamente alla riduzione delle emissioni di gas serra. Nonostante le bassissime emissioni e i costi energetici significativamente ridotti rispetto all'impiego di combustibili fossili, la diffusione dei sistemi che sfruttano l'energia geotermica a bassa entalpia (profondità generalmente comprese tra i $30 \mathrm{e}$ i $150 \mathrm{~m}$ dal piano campagna), è ancora limitata da molteplici fattori. In questo ambito, gli amministratori del territorio esercitano un ruolo chiave in quanto, attraverso la predisposizione di strumenti di vario tipo (informativi, normativi e tecnici), essi possono imprimere un impulso importante allo sviluppo dei sistemi geotermici di bassa profondità, ampliando così il ventaglio di tecnologie attualmente disponibili per lo sfruttamento delle fonti energetiche rinnovabili.

Il progetto Interreg GRETA (Near-surface Geothermal Resources in the Territory of the Alpine Space), partendo dalla condivisione di esperienze, di linee guida e di buone pratiche osservate nei paesi dello Spazio Alpino (Figura 1), ha l'obbiettivo di formulare proposte per l'implementazione di strumenti normativi armonizzati, di produrre mappe di potenziale e di proporre approcci per l'integrazione della geotermia di bassa profondità nei piani energetici comunali e regionali.

Nell'ambito di questo progetto, Regione Lombardia (partner del progetto GRETA) ha organizzato un workshop con lo scopo di condividere con gli amministratori del territorio i primi risultati e di illustrare i prossimi sviluppi del progetto. L'evento ha visto il coinvolgimento di Amministrazioni Regionali e Provinciali dello Spazio Alpino italiano e di diversi altri enti presenti sul territorio (ARPA, Ordini dei Geologi, etc.), con la partecipazione di circa quaranta persone. Erano inoltre presenti rappresentanti di alcuni partner stranieri del progetto: l'Università Tecnica di Monaco di Baviera (TUM), il Servizio Geologico Sloveno (Geo-ZS) e quello austriaco (GBA). Si rimarca come l'evento abbia rappresentato una novità nel panorama formativo ed informativo sulla materia, essendo il primo in Italia destinato specificatamente agli amministratori del territorio: tipicamente, eventi di questo tipo sono destinati a tecnici o ad imprese del settore. La modalità organizzativa dell'evento ha permesso di instaurare discussioni tematiche affrontate attraverso gruppi di lavoro, finalizzate specificamente al tentativo di instaurare una convergenza tra diversi enti, cercando quindi di superare la frammentazione che si osserva tra i diversi ambiti territoriali in tema di normativa e di procedure autorizzative sugli impianti geotermici di bassa entalpia.

I temi di discussione affrontati dai gruppi di lavoro sono stati: i) l'armonizzazione della normativa, ii) le banche dati e le mappe WebGis e iii) le procedure "a sportello unico".

\section{Presentazione del workshop}

Ad introduzione del workshop sono intervenuti alcuni dei partner stranieri del Progetto GRETA, citati poc'anzi: TUM, partener leader del progetto, ha fatto una presentazione introduttiva del progetto, dopodiché Geo-ZS, responsabile della 




Fig.1: - Area dello Spazio Alpino europeo.

Fig. 1: Area of the European Alpine Space.

parte di progetto riguardante la normativa di settore, ha fornito una panoramica sulla situazione legislativa dei sistemi geotermici nei paesi dello Spazio Alpino. Similmente, GBA, che è partner anche del progetto Interreg GEOplasma per lo sviluppo della geotermia in Europa Centrale, ha fornito una panoramica sul quadro normativo dei sistemi geotermici di bassa profondità in Europa Centrale; cfr. https://www.interreg-central.eu/Content.Node/GeoPLASMA-CE.html).

Dalle presentazioni è emerso che la frammentazione della normativa specifica sulla geotermia di bassa profondità è una caratteristica presente in tutti i Paesi europei menzionati. Questa frammentazione, che in Italia è generata dalla presenza di procedure definite in maniera locale da Regioni e Province, non è quindi un aspetto esclusivamente italiano. Tuttavia, è emerso anche che la frammentazione della normativa non rappresenta necessariamente un ostacolo alla diffusione di questi impianti, nei casi in cui le procedure autorizzative sono chiaramente definite e le informazioni tecniche e normative sono facilmente reperibili attraverso portali web.

Gli altri partner del progetto GRETA hanno illustrato i risultati preliminari del progetto, evidenziando la multidisciplinarietà degli argomenti trattati: la compilazione di un catalogo di procedure tecniche e di "buone pratiche", la predisposizione di carte di potenziale geotermico a grande e piccola scala, l'implementazione di strumenti utili alla definizione dei costi di installazione e alla pianificazione energetica. I risultati del progetto e gli strumenti elaborati sono disponibili sul portale Cesba Wiki all'indirizzo http://wiki.cesba.eu/ wiki/GRETA.

Non è mancata poi una panoramica sulla situazione italiana, incentrata in particolare sulla Regione Lombardia, che può vantare una normativa più dettagliata e propositiva rispetto a molte altre regioni italiane. Prima della discussione dei temi dell'armonizzazione normativa, delle banche dati e mappe WebGis e delle procedure "a sportello unico", è stata of- ferta agli enti territoriali partecipanti l'opportunità di presentare le peculiarità del territorio di appartenenza, specificando $i$ punti di forza e i punti di debolezza delle procedure autorizzative specifiche della propria Regione. In questa occasione, sono emerse una serie di aspetti migliorabili e di ostacoli da superare nell'ottica di una maggiore diffusione di queste tecnologie.

\section{Punti di forza e punti di debolezza italiani}

All'inizio del workshop è stato chiesto ai partecipanti di presentare brevemente la situazione normativa nel proprio ambito territoriale, specificando i punti ritenuti maggiormente positivi e quelli, invece, da migliorare.

Dalla discussione è emerso che l'assenza di una normativa nazionale chiara ed univoca è uno degli handicap più grandi per lo sviluppo della geotermia a bassa entalpia in Italia. Sono tuttavia stati evidenziati alcuni casi virtuosi, come:

- Il regolamento della Regione Lombardia RR 7/10, che introduce il catasto delle sonde geotermiche e impone l'obbligo di registrazione dei nuovi impianti nello stesso catasto, utile per il monitoraggio in tempo reale della diffusione degli impianti a circuito chiuso unitamente agli indirizzi tecnici contenuti nella d.g.r. 6203/17 per la reimmissione in falda di acque sotterranee prelevate per scambio termico. Quest'ultimo provvedimento rappresenta una razionalizzazione delle procedure amministrative concessorie/autorizzatorie per impianti a circuito aperto di piccola taglia;

- La normativa riguardo ai sistemi a circuito aperto del Friuli-Venezia-Giulia, completa di modulistica chiara e di un censimento degli impianti;

- La situazione della provincia Autonoma di Trento, la quale, per i sistemi a circuito chiuso, dispone di una normativa specifica, una modulistica dedicata e offre un web-GIS per l'individuazione delle aree ove è consentita l'installazione degli impianti. 




Fig.2: Il programma del workshop.

Fig. 2: Program of the workshop.

Sono state, inoltre, evidenziate alcune criticità:

- In Friuli-Venezia-Giulia, per i sistemi a circuito aperto, si riscontra la difficoltà nell'effettuare i controlli e l'assenza di obblighi di monitoraggio; mentre per i sistemi a circuito chiuso, si evidenzia la mancanza di una normativa specifica;

- In Valle d'Aosta si evidenzia da un lato l'assenza di una precisa normativa che regolamenti gli impianti a circuito chiuso, dall'altro si sottolinea la presenza di una norma del PTA vigente che vieta la reimmissione in falda delle acque prelevate per usi geotermici, vincolando di fatto la realizzazione di impianti a circuito aperto alla possibilità di disporre di un corpo idrico superficiale adiacente all'impianto in cui scaricare le acque emunte.

- Nella provincia Autonoma di Trento non sono di fatto attuati i controlli sugli impianti a circuito chiuso; inoltre la modulistica sarebbe da migliorare;

- In Regione Lombardia, per quanto riguarda gli impianti a circuito chiuso, non è ancora stato attivato l'obbligo di effettuare il monitoraggio in esercizio, che risulterebbe invece necessario per gli impianti di grande taglia. Inoltre, poiché le profondità consentite sono molto elevate, emerge la necessità di linee guida per la corretta esecuzione del campo sonde geotermico, e una normativa che definisca la necessità della direzione lavori specialistica per la verifica del modello geologico - idrogeologico individuato nella fase progettuale.



Fig.3: Presentazione introduttiva del Progetto GRETA.

Fig.3: Introductive presentation of the GRETA Project.

\section{Armonizzazione della normativa}

La necessità di prevedere un'armonizzazione della normativa è emersa con forza durante la discussione all'interno del gruppo di lavoro dedicato. Nonostante in altri contesti europei la disomogeneità della normativa non abbia rappresentato un impedimento alla diffusione della tecnologia (ad esempio in Baviera), l'armonizzazione appare necessaria per diverse ragioni:

- una normativa omogenea permette di definire meglio i tempi necessari per le autorizzazioni su tutto il territorio;

- una normativa omogenea rappresenta uno strumento di equità nei confronti dei cittadini, fornendo le stesse opportunità per accedere alla tecnologia indipendentemente dalla regione di residenza;

- considerando la riduzione delle emissioni di CO2 ottenibile mediante l'uso di questa tecnologia, una normativa omogenea rappresenta uno strumento di omogeneizzazione delle politiche per la riduzione dei gas serra e per la promozione dello sviluppo sostenibile;

- una normativa omogenea permette una uniforme e migliore tutela dell'ambiente e in particolare degli acquiferi.

Una nuova a normativa di settore dovrebbe quindi definire in maniera omogenea:

- quali siano gli enti pubblici designati al disbrigo delle pratiche autorizzative, individuando al contempo i compiti dei vari enti;

- quali siano i compiti dei progettisti, individuando in particolare le categorie professionali competenti, distinguendo la parte puramente impiantistica dalla parte di valutazione geologica sull'interazione dell'opera con il sottosuolo;

- le regole e i vincoli amministrativi, ambientali e tecnici;

- $\quad$ quali siano le informazioni da acquisire in fase di progettazione e quelle da acquisire a seguito dell'installazione e durante il funzionamento dell'impianto;

- $\quad$ quali siano le specifiche e le procedure per il censimento degli impianti geotermici in apposite banche dati, come previsto dall'art. 2 del D.Lgs. 22/2010; 
- quali debbano essere le competenze e i compiti della Direzione Lavori in cantieri per l'installazione di sistemi geotermici. Queste devono comprendere l'assistenza del geologo per l'acquisizione dei dati geologico-ambientali da inserire nella banca dati di cui al punto precedente;

- quali siano le metodologie di attuazione dei controlli, individuando gli enti preposti e le tempistiche;

- quali siano le sanzioni per il mancato rispetto delle norme o per i danni ambientali che potrebbero essere indotti da una non corretta installazione dell'impianto: in questo senso viene proposta l'introduzione dell'obbligo di stipulare una fideiussione, almeno per gli impianti di una certa dimensione;

- una procedura autorizzativa semplificata per gli impianti a circuito chiuso, in funzione della potenza installata;

- $\quad$ per gli impianti a circuito aperto, la normativa dovrebbe introdurre in maniera omogenea l'obbligo di simulazioni sugli effetti quantitativi e qualitativi degli impianti sulla falda, particolarmente dettagliati in aree ad alta densità abitativa o in presenza di zone di tutela dell'acquifero (es pozzi idropotabili).

Per raggiungere l'obbiettivo di una unificazione delle procedure, che permetta di snellire la burocrazia e al contempo tuteli le risorse idriche sotterranee, appare necessario:

- introdurre lo 'sportello unico' per la presentazione delle procedure;

- introdurre una distinzione tra procedure autorizzative per impianti di "piccola taglia" e "impianti di grossa taglia” (come in parte già menzionato nel D.Lgs. 22/2010;

- il recepimento della Direttiva Europea 2009/28/CE, e l'emanazione di una normativa specifica sui sistemi geotermici a bassa entalpia a livello nazionale;

- $\quad$ prevedere la possibilità, da parte di Regioni e Province Autonome, di recepire la normativa specifica a livello nazionale, adattandola al contesto territoriale con specificazioni locali, che non si pongano in contrasto con le procedure di unificazione delle procedure proposte a livello nazionale;

- corredare allegati tecnici condivisi con gli Ordini Professionali, in particolare con Geologi e Ingegneri, ed eventualmente redigere Linee Guida Nazionali concordate con questi Ordini Professionali.

\section{Banche dati e mappe WebGIS}

Dalla discussione di questo gruppo di lavoro è emerso che l'effettiva utilità della realizzazione di banche dati e WebGIS specifici sulla geotermia di superficie è legata ad una corretta definizione delle finalità di questi strumenti, in funzione degli utenti.

I professionisti devono poter trovare nei WebGIS e nei database strumenti utili per progettare e promuovere la diffusione della tecnologia, per una migliore integrazione tra questa fonte energetica e le altre fonti rinnovabili a disposizione del cliente finale, per pianificare il monitoraggio post-installazione nella maniera più efficace, e per migliorare l'interpretazione dei dati raccolti dal database stesso.

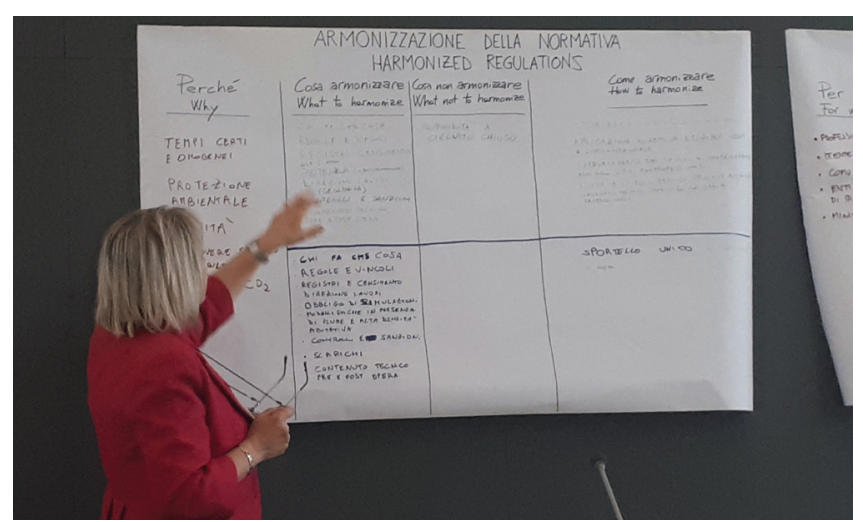

Fig.4: Presentazione dei risultati del gruppo di lavoro.

Fig. 4: Presentation of the results of the discussion groups.

Tra i principali utilizzatori di questi strumenti sono individuati i Comuni e più in generale gli Enti locali, per poter integrare la geotermia a bassa entalpia nella pianificazione energetica territoriale, e conseguentemente promuovere la divulgazione delle opportunità fornite da questa fonte rinnovabile. I database possono, inoltre, rappresentare uno strumento per questi enti per vigilare sul rispetto della normativa per quanto concerne i limiti termici definiti in fase di progetto, e per verificare l'effettiva attuazione del monitoraggio.

Gli Enti nazionali, quali ad esempio i Ministeri dell'Ambiente e dell'Economia, possono utilizzare i dati contenuti nei database e WebGIS per predisporre gli strumenti di pianificazione energetica nazionale, e per evidenziare le specificità della risorsa allo scopo di favorirne l'integrazione con le altre fonti energetiche.

Anche gli Enti di Ricerca potrebbero fruire di questi dati (ad esempio quelli acquisiti mediante i monitoraggi) al fine di fornire strumenti per la pianificazione energetica.

Inoltre, questi strumenti rappresentano una importante fonte di divulgazione e permettono ai cittadini di acquisire informazioni per decidere se prediligere questa tecnologia ad altre disponibili.

Per raggiungere tutti gli obbiettivi prefissati, le informazioni contenute all'interno della banca dati dovrebbero innanzi tutto riguardare gli impianti esistenti e quelli di nuova installazione. Ad ogni impianto caricato nel webGIS, occorrerebbe associare almeno le seguenti informazioni:

- tipologia dell'impianto, potenza, utilizzo (riscaldamento e/o raffrescamento);

- $\quad$ tipo di utenza servito;

- $\quad$ stratigrafie e soggiacenze acquisite in corso di realizzazione dell'impianto;

- caratteristiche della falda (soggiacenza, temperatura, classificazione ai sensi del D.Lsg. 30/2009);

- dati costruttivi dei pozzi;

- $\quad$ portate e volumi emunti.

Tra i tematismi del WebGIS, oltre a quelli a carattere geologico-idrogeologico, generalmente già presenti nei principali Geoportali territoriali, sarebbe utile elaborare ed inserire le carte del potenziale geotermico. 
Per una migliore fruizione e diffusione dello strumento, la struttura del WebGIS e della banca dati dovrebbe ricalcare per quanto possibile quella degli analoghi strumenti già esistenti a carattere regionale o nazionale, uniformandosi per quanto possibile con la Direttiva Inspire (recepita in Italia con il D.Lgs. 32/2010). Inoltre, è auspicabile il collegamento diretto con questi stessi Geoportali, in modo da favorire l'interpretazione dei dati e l'utilizzo ragionato degli stessi.

\section{Procedure a 'sportello unico'}

Con il termine "procedure a sportello unico" o "one-stop shop", si intende la semplificazione dell'iter autorizzativo, prevedendo non tanto una riduzione degli elaborati progettuali quanto la riduzione del numero di enti ed uffici di competenza a cui occorre presentare documenti o richieste autorizzative, nell'ottica di una riduzione dell'incertezza dei tempi autorizzativi. Nel gruppo di lavoro dedicato a questa tematica, la necessità di individuare procedure semplificate per ottenere le autorizzazioni è emersa in maniera indiscussa, indipendentemente dalla tipologia di impianti geotermici.

Per quanto riguarda gli impianti a circuito chiuso, dato il loro minimo impatto ambientale, non dovrebbero essere previsti vincoli legati alle dimensioni degli impianti. Nella discussione è emerso il modello di Regione Lombardia, ove per questa tipologia di impianti lo snellimento delle procedure già attuato dal 2011 sembra avere raggiunto l'obiettivo di far crescere significativamente il mercato della geotermia a bassa entalpia. In questa regione, infatti, la realizzazione degli impianti a circuito chiuso è permessa, fino ad una profondità massima di $150 \mathrm{~m}$, senza necessità di richiesta autorizzativa, attraverso la semplice registrazione dell'impianto nel Registro delle Sonde Geotermiche.

Per quanto riguarda i sistemi a circuito aperto, l'interazione attiva con la falda pone la necessità di definire delle norme più cautelative. L'elaborazione di procedure semplificate, quindi, dovrebbe essere mirata a impianti di dimensioni mediopiccole, definendo un limite sulla base della portata emunta.



Fig.5: Gruppi di discussione al lavoro.

Fig. 5: Discussion groups at work.
Il principale aspetto critico a livello autorizzativo appare essere quello della fase di re-immissione in falda delle acque emunte. In sostanza si propone di considerare questa operazione come una restituzione, invece dell'attuale interpretazione che la considera uno scarico e come tale ne prescrive le procedure autorizzative. In questo modo, le concessioni di prelievo e di restituzione diventerebbero un procedimento unico, permettendo:

- la riduzione del numero di uffici competenti a cui chiedere l'autorizzazione;

- la semplificazione della procedura e la riduzione dei tempi di risposta;

- l'applicazione di un'unica durata della concessione valida sia che per la restituzione.

In generale, per una maggior diffusione della tecnologia, emerge la necessità di individuare incentivi economici, di raccogliere ed elaborare statistiche sugli impianti già esistenti, e di sviluppare database appositi.

\section{Conclusioni}

Nella parte finale del workshop sono stati sintetizzati i risultati emersi dai gruppi di lavoro e per condividere le strategie da intraprendere per dare maggiore impulso alla diffusione dei sistemi basati sullo sfruttamento della geotermia di bassa profondità. In sintesi, appare urgente innanzi tutto:

- la redazione di una normativa specifica a livello nazionale, da recepire da parte di Regioni e Province Autonome attraverso norme e linee guida che la adattino al contesto territoriale;

- la stretta collaborazione tra Ordini Professionali, in particolare con Geologi e Ingegneri, e i redattori delle normative e delle linee guida nazionale e regionali;

- la creazione, in ogni Regione, di banche dati con le informazioni geologiche e impiantistiche degli impianti esistenti e in costruzione. Sarebbe auspicabile che le diverse banche dati fossero convogliate su una banca dati unica nazionale;

- la creazione sul territorio nazionale di un WebGis con le carte del potenziale geotermico;

- la semplificazione delle procedure per gli impianti a circuito chiuso e aperto di piccola taglia;

- la definizione di un unico ente/ufficio competente per la richiesta di autorizzazione per gli impianti a circuito aperto;

- la necessità di individuare incentivi economici specifici più importanti rispetto a quelli attualmente esistenti che riguardano solo alcune parti dell'impianto (pompa di calore).

Più in generale, si rimarca come questo evento abbia rappresentato l'occasione per avviare un confronto costruttivo tra amministratori del territorio, coinvolgendo anche i professionisti e gli Ordini Professionali. È emersa in maniera condivisa la necessità di riproporre analoghe occasioni di incontro tra gli amministratori del territorio, nell'ottica di trovare convergenze sullo sviluppo di normative e linee guida specifiche e di strumenti utili per la promozione dell'utilizzo di impianti geotermici che sfruttano la geotermia di bassa profondità. 